available data was carried out by in-country 'informed lay persons'.

Results: Three main messages emerged. First, robust evidence-based population and policy indicators could be postulated related to determinants of obesity. Second, there is a dearth of available data. Attempting to populate the indicators showed many statistics were not scientifically optimum or consistent across Europe. Data were often absent, incomplete or collected as a one-off activity. Even countries with comprehensive data surveillance could not populate all indicators. Third, there is more emphasis on post hoc measurement of obesity than on determinants.

Conclusions: National data about children needs to be prioritised. Collecting data at key points, policy analysis and routine analysis from a child-centric perspective of, e.g., census and survey data would yield a clearer picture on behavioural determinants of health and conversely, obesity. At present thorough scientific analysis is compromised and evidence-based policy making very difficult. An indicator set based on potentially available data is identified and feasible.

Funding: Research relating to this abstract was funded by the Directorate General Health and Consumer Protection of the European Commission - the overall project was run by the Technical University of Dresden; the work on children was scientifically led by Professor Rigby at Keele University and coordinated by Denise Alexander.

\title{
80 - Body dissatisfaction in Portuguese adolescents: gender and obesity prevalence
}

\author{
E Coelho, L Oliveira, S Fonseca and I Mourao-Carvalhal \\ CIDESD, University of Tras-os-Montes e Alto Douro, Portugal
}

Introduction: The aim of the present study was to compare body dissatisfaction in Portuguese adolescents by gender and obesity prevalence.

Method: A cross-sectional study including 353 adolescents (167 males, 78 females) aged 12-19 years (16.25 (sD 1·36)). The prevalence of obesity was calculated by BMI and used the cut-offs for overweight and obesity defined by Cole et al. (2000). Body image was assessed by a range of seven silhouettes (Stunkard et al. 1983) and body dissatisfaction was estimated by the difference between current and ideal body image. Chi-square test was used to compare groups.

Results: Significant differences was found between gender $\left(X^{2}=50 \cdot 488, P=0 \cdot 000\right)$. A greater proportion of girls perceived themselves as fat $(70 \cdot 6 \% v .26 \%)$ and a higher percentage of boys perceived themselves as thin $(74 \% \mathrm{v}$. $26 \%$ ). Differences in body dissatisfaction according to the prevalence of obesity were statistically significant in both gender (male: $X^{2}=42 \cdot 636, P=0 \cdot 000$; female: $X^{2}=17 \cdot 658$, $P=0 \cdot 000$ ). For males, $17 \cdot 5 \%$ of normal weight perceived themselves as fat, $29 \cdot 2 \%$ were satisfied with their image and $53.3 \%$ perceived themselves as thin. For obese (overweight + obese), $76 \cdot 7 \%$ perceived themselves as fat, $10 \%$ were satisfied with their image and $13.3 \%$ perceived themselves as thin. For females, $54.4 \%$ of normal weight perceived as being fat, $28.5 \%$ were satisfied with their image and $17 \cdot 1 \%$ perceived themselves as being thin. For the obese, $96 \cdot 4 \%$ perceived as obese and 3.6\% were satisfied with their image.

Conclusions: Gender and obesity prevalence are variables that differentiate body image dissatisfaction. The female adolescents see themselves as being fatter compared with male adolescents, who perceived themselves as being thinner. The standards of beauty imposed by our society for girls may justify their greater body dissatisfaction.

\section{1 - Does body image self-perception matches reality in elementary-school children?}

\section{G Leduc $^{1,2}, \mathrm{~S}$ Dodin $^{3,4}, \mathrm{C} \mathrm{Blanchet}^{3,4}$ and N Almeras ${ }^{1}$}

\footnotetext{
${ }^{1}$ Institut Universitaire de Cardiologie et de Pneumologie de Quebec Research Center, Quebec, Canada: ${ }^{2}$ Department of Food Sciences and Nutrition, Universite Laval, Quebec, Canada: ${ }^{3}$ Department of Obstetrics and Gynecology, Research Center, St-Francois d'Assise Hospital (CHUQ), Quebec, Quebec, Canada: ${ }^{4}$ Institute of Nutraceuticals and Functional Foods, Universite Laval, Quebec, Quebec, Canada
} 
Introduction: Studies showed that many adolescents and adults misperceive their weight status. Fewer data exist on this topic regarding children. The objective of the present study was to evaluate the concordance between BMI Z-score (BMIZ) and body image self-perception (BISP) in elementary school-aged children.

Method: The present study included 262 children (9.2 (sD 1.6) years) from the Quebec City suburbs. Children selected their BISP using Collin's pictorial scale. The seven body shapes were recodified to -3 (very thin) to 3 (very obese) so that the body shape representing an average-weight child was numbered 0 and was associated to a BMIZ of 0. Children's height and weight were measured. They were considered normal or overweight/ obese if presenting a $\mathrm{BMIZ}<1$ or $\geq 1$, respectively.

Results: Twenty-seven per cent of children could accurately auto-evaluate their body shape, while $72 \%$ were inaccurate (59\% underestimated, 13\% overestimated). After grouping children according to their age $(<9 \quad v . \geq 9$ years old) and BMIZ $(<1 v$. $\geqslant 1)$, younger children presented a greater difference between BMIZ and BISP than older ones $(P<0 \cdot 05)$. Irrespective of age, normal weight and overweight/obese children perceived themselves thinner than their actual BMIZ (sBISP/ $\mathrm{BMIZ}=-0.50(\mathrm{SD} 1 \cdot 04) v .-1 \cdot 50$ (SD 0.86), respectively; $P<0 \cdot 001)$. Interestingly, on average, both normal and overweight/obese children perceived themselves as showing an average BMIZ.

Conclusions: The present study showed that a large majority of children are inaccurate in auto-evaluating their body image, which is especially striking with overweight and obese children. Further studies might explore whether this component should be part of obesity prevention programmes involving children.

\title{
82 - Are elementary school-aged children satisfied with their body image?
}

\author{
G Leduc $^{1,2}, \mathrm{SDodin}^{3,4}, \mathrm{C}$ Blanchet $^{3,4}$ and N Almeras ${ }^{1}$ \\ ${ }^{1}$ Institut Universitaire de Cardiologie et de Pneumologie de Quebec Research Center, Quebec, \\ Canada: ${ }^{2}$ Department of Food Sciences and Nutrition, Universite Laval, Quebec, Canada: \\ ${ }^{3}$ Department of Obstetrics and Gynecology, Research Center, St-Francois d'Assise Hospital \\ (CHUQ), Quebec, Canada: ${ }^{4}$ Institute of Nutraceuticals and Functional Foods, Universite Laval, \\ Quebec, Quebec, Canada
}

Introduction: Dissatisfaction with body image might become a major issue with the increasing prevalence of obesity among children. The objectives of the present study were to document the relationships between body image self-perception (BISP) and ideal body image (IBI) in elementary school-aged children.

Method: The present study included 262 children $(9 \cdot 2$ (sD 1.6) years) from the Quebec City suburbs. Children selected their BISP and IBI using Collin's pictorial scale. The seven body shapes were re-codified to -3 (very thin) to 3 (very obese) so that the body shape representing an average-weight child was numbered 0 and was associated to a BMIZ of 0. Children's height and weight were measured. They were considered normal or overweight/obese if presenting a BMIZ $<1$ or $\geq 1$, respectively.

Results: Fifty-three per cent of children did not wish to have a different body image than what they perceived,
$36 \%$ wished to be thinner and $12 \%$ wished to be heavier. Children presented a BMIZ of $0 \cdot 17$ (SD 0.91) with a BISP of -0.53 (SD 0.97 ) and an IBI of -0.84 (SD 1.03). BISP was significantly lower than BMIZ and IBI was even significantly lower than BISP $(P<0 \cdot 001)$. IBI was not different between boys $(-0.74$ (SD 1.07)) and girls $(-0.92(\mathrm{SD} 1 \cdot 00))$. Moreover, even though normal and overweight/obese children were different for BISP $(-0.65$ (SD 0.96) $v . \quad-0.04 \quad$ (SD 0.82), respectively; $P<0 \cdot 001)$, their IBI were similar $(-0 \cdot 8(\mathrm{SD} 1 \cdot 0) v .-1 \cdot 0$ (sD $1 \cdot 2$ ), respectively; ns).

Conclusions: One out of two children was satisfied with his/her perceived body image. It is noteworthy that children's ideal body image seemed to be the same, no matter what their gender or weight status were.

Funding: Centre de recherche en prevention de l'obesite (CRPO); Fondation Lucie et Andre Chagnon; Fonds de la recherche en sante du Quebec (FRSQ). 